\title{
DETERMINATION OF OPTIMAL UNIT HYDROGRAPHS BY LINEAR \\ PROGRAMMING
}

\author{
Bing Zhao \\ and \\ Yeou-Koung Tung \\ Journal Article $1994 \quad$ WWRC-94-24
}

In

Water Resources Management

Volume 8

\author{
Bing Zhao \\ Department of Civil Engineering \\ Arizona State University \\ Tempe, Arizona \\ Yeou-Koung Tung \\ Wyoming Water Resources Center \\ and \\ Statistics Department \\ University of Wyoming \\ Laramie, Wyoming
}




\title{
Determination of Optimal Unit Hydrographs by . Linear Programming
}

\author{
BING ZHAO \\ Department of Civil Engineering, Arizona State University Tempe, AZ 85287, U.S.A. \\ and \\ YEOU-KOUNG TUNG \\ Wyoming Water Resources Center and Statistics Department, University of Wyoming, Laramie, WY \\ 82071, U.S.A.
}

(Received 11 March 1993; accepted in final form: 20 May 1994)

\begin{abstract}
A unit hydrograph (UH) obtained from past storms can be used to predict a direct runoff hydrograph (DRH) based on the effective rainfall hyetograph (ERH) of a new storm. The objective functions in commonly used linear programming (LP) formulations for obtaining an optimal UH are (1) minimizing the sum of absolute deviations (MSAD) and (2) minimizing the largest absolute deviation (MLAD). This paper proposes two alternative LP formulations for obtaining an optimal UH, namely, (1) minimizing the weighted sum of absolute deviations (MWSAD) and (2) minimizing the range of deviations (MRNG), In this paper the predicted DRHs as well as the regenerated DRHs by using the UHs obtained from different LP formulations were compared using a statistical crossvalidation technique. The golden section search method was used to determine the optimal weights for the model of MWSAD. The numerical results show that the UH by MRNG is better than that by MLAD in regenerating and predicting DRHs. It is also found that the model MWSAD with a properly selected weighing function would produce a UH that is better in predicting the DRHs than the commonly used MSAD.
\end{abstract}

Key words: unit hydrograph, linear programming, validation.

\section{Notations}

$M=$ number of effective rainfall increments.

$N=$ number of direct runoff hydrograph ordinates.

$R=$ number of storms.

MSAD $=$ minimize sum of absolute deviation.

MWSAD $=$ minimize weighted sum of absolute deviation.

MLAD $=$ minimize the largest absolute deviation.

MRNG $=$ minimize the range of deviation.

RMSE $=$ root mean square error.

$P_{m}=$ effective rainfall in time interval $[(m-1) \Delta t, m \Delta t]$.

$Q_{n}=$ direct runoff at discrete time $n \Delta t$.

$U_{k}=$ unit hydrograph ordinate at discrete time $k \Delta t$.

$W_{n}=$ weight assigned to error associated with estimating $Q_{n}$.

$\varepsilon_{n}^{+}=$error associated with over-estimation of $Q_{n}$.

$\varepsilon_{n}^{-}=$error associated with under-estimation of $Q_{n}$. 


$$
\begin{aligned}
\varepsilon^{+}{ }_{\max } & =\text { maximum positive error in fitting direct runoff hydrograph. } \\
\varepsilon^{-}{ }_{\max } & =\text { maximum negative error in fitting direct runoff hydrograph. } \\
\varepsilon_{\max } & =\text { largest absolute error in fitting obtained direct runoff. } \\
E_{r, 1} & =\text { the } l \text { th error criterion measuring the fit between the observed } \\
E_{1} & =\text { DRHs and the predicted (or reproduced) DRHs for the } r \text { th storm. }
\end{aligned}
$$

\section{Introduction}

The unit hydrograph (UH) proposed by Sherman (1932) is defined as a direct runoff hydrograph (DRH) resulting from one unit of effective rainfall distributed uniformly over a watershed for a specified duration. In UH theory, the watershed is considered as a system with effective rainfall hyetograph (ERH) being the input, DRH being the output, and UH being the kernel function. The discrete convolution relationship that relates the ERH, DRH, and $\mathrm{UH}$ can be written as (Chow et al., 1988).

$$
Q_{n}=\sum_{m=1}^{n \leq M} P_{m} U_{n-m+1},
$$

in which $P_{m}$ is the $m$ th ordinate of the ERH $(m=1,2,3, \ldots, M)$ with $M$ being the number of ERH ordinates, $Q_{n}$ is the $n$th ordinate of the $\mathrm{DRH}(n=1,2,3 \ldots, N)$ with $N$ being the number of DRH ordinates, and $U_{r}$ is the $r$ th $\mathrm{UH}$ ordinates characterizing the rainfall-runoff relation for the watershed. The total number of UH ordinates is $N-M+1$. Given the ERH and DRH obtained from a storm occurring in a watershed, Equation (1) can be used to derive the corresponding UH. The derived UH, in turn, can be used to predict the DRH of a storm if the ERH is known.

There have been many methods developed for deriving UHs from complex storm events which include successive approximations (Collins, 1939; Barnes, 1959; Bender and Roberson, 1961), the system transformations (O'Donnel, 1960; Dooge, 1965; Dooge and Garvey, 1978), least-squares and its variations (Snyder, 1955; Newton and Vinyard, 1967; Kuchment, 1967; Singh, 1976; Bruen and Dooge, 1984), and linear programming (Deininger, 1969; Eagleson et al., 1966; Singh, 1976; Mays and Coles, 1980; Morel-Seytoux, 1982; Singh, 1988).

In a previous study on the UH determination using the linear programming (LP) approach, two types of objective functions have been used: (1) minimization of the sum of absolute deviations (MSAD) and (2) minimization of the largest absolute deviation (MLAD). The LP formulation based upon the MSAD criterion can be stated as

minimize

$$
\varepsilon_{1}=\sum_{n=1}^{N}\left(\varepsilon_{n}^{+}+\varepsilon_{n}^{-}\right)
$$


subject to

$$
\begin{aligned}
& \sum_{m=1}^{n \leq M} P_{n} U_{n-m+1}-\varepsilon_{n}^{+}+\varepsilon_{n}^{-}=Q_{n}, \quad n=1,2, \ldots, N, \\
& \Delta t \sum_{r=1}^{N-M+1} U_{r}=1, \\
& U_{r} \geq 0, r=1,2, \ldots, N-M+1 \\
& \varepsilon_{n}^{+} \geq 0, \varepsilon_{n}^{-} \geq 0, \quad n=1,2, \ldots, N,
\end{aligned}
$$

in which $\varepsilon_{n}{ }^{+}$and $\varepsilon_{n}{ }^{-}$are nonnegative decision variables representing errors due to over-estimation and under-estimation, respectively; and $\Delta t$ is the UH duration. The units for $P_{m}, Q_{n}$, and $U_{r}$ used in this formulation are $\mathrm{mm}, \mathrm{mm} / \mathrm{h}$, and $1 / \mathrm{h}$, respectively. For the above model (Equations (2a)-(2d)), the number of decision variables is $3 N-M+1$ and the number of constraints is $N+1$.

The LP model based upon the MLAD criterion can be formulated as

minimize

$$
\varepsilon_{2}=\varepsilon_{\max }
$$

subject to

$$
\begin{aligned}
& \sum_{m=1}^{n \leq M} P_{n} U_{n-m+1}-\varepsilon_{\max } \leq Q_{n}, \quad n=1,2, \ldots, N, \\
& \sum_{m=1}^{n \leq M} P_{n} U_{n-m+1}+\varepsilon_{\max } \geq Q_{n}, \quad n=1,2, \ldots, N, \\
& \Delta t \sum_{r=1}^{N-M+1} U_{r}=1, \\
& U_{r} \geq 0, \quad r=1,2, \ldots, N-M+1 ; \quad \varepsilon_{\max } \geq 0,
\end{aligned}
$$

in which $\varepsilon_{\max }$ is a nonnegative decision variable representing the largest absolute deviation. The number of decision variables in this LP formulation is $N-M+2$, which is significantly less than that in the MSAD formulation, while the number of constraints is $2 N+1$, which is about twice as many as that in the MSAD.

The purposes of this paper are (1) to propose two alternative LP formulations for determining optimal UHs and (2) to evaluate the relative performance 
of derived UHs from the various LP formulations. More specifically, statistical cross-validation technique was employed in the investigation. The various LP formulations were applied to 20 storms in Bree's paper (1978). The two proposed alternative LP formulations for the UH determination are (1) the minimization of the weighted sum of absolute deviations (MWSAD) and (2) minimization of the range of deviations (MRNG). The MSAD formulation is a special case of the MWSAD model, since the MSAD criterion assigns equal weights to all deviations in the objective function. Instead of controlling the largest absolute deviation as in the MLAD formulation, the MRNG criterion controls the range of deviations.

Since the optimal UH obtained from minimizing one form of objective function may not be the optimal UH for predicting other storms or future storms, it is essential to examine the predictability of UHs obtained from different LP formulations. The predictability of UHs derived from the MSAD, MLAD, MWSAD, and MRNG was assessed by applying the derived UHs to other observed storms using a statistical cross-validation technique. The MWSAD formulation with different values of the weighing function were considered. The golden section search method was applied to determine the optimal weights for the MWSAD criterion that yields the best predictability.

\section{Minimizing the Weighted Sum of Absolute Deviations (MWSAD)}

The LP formulation based upon the MWSAD criterion can be mathematically expressed as

minimize

$$
\varepsilon_{3}=\sum_{n=1}^{N} W_{n}\left(\varepsilon_{n}^{+}+\varepsilon_{n}^{-}\right),
$$

subject to

$$
\begin{aligned}
& \sum_{m=1}^{n \leq M} P_{n} U_{n-m+1}-\varepsilon_{n}^{+}+\varepsilon_{n}^{-}=Q_{n}, \quad n=1,2, \ldots, N, \\
& \Delta t \sum_{r=1}^{N-M+1} U_{r}=1, \\
& U_{r} \geq 0, r=1,2, \ldots, N-M+1 \\
& \varepsilon_{n}^{+} \geq 0, \varepsilon_{n}^{-} \geq 0, \quad n=1,2, \ldots, N,
\end{aligned}
$$

in which $W_{n}$ is the weight assigned to the deviation of the $n$th DRH ordinate. The weighing function considered in this study is

$$
W_{n}=\frac{N Q_{n}^{\alpha}}{\sum_{n=1}^{N} Q_{n}^{\alpha}}, \quad n=1,2, \ldots, N
$$


in which $\alpha$ is a constant. Note that when $\alpha=0$, the MWSAD formulation reduces to the MSAD model. As a common practice in hydrology, one often uses positivevalued $\alpha$ in Equation (5). The larger the value of $\alpha$, the more emphasis is given to fit closely to higher discharge values.

\section{Minimizing the Range of Deviations (MRNG)}

The range of deviation is defined as $\varepsilon_{\mathrm{mg}}=\varepsilon_{\max }{ }^{+}+\varepsilon_{\max }{ }^{-}$with $\varepsilon_{\max }{ }^{+}=\max \left\{\varepsilon_{n}{ }^{+}\right\}$, the largest value of over-estimation, and $\varepsilon_{\max }{ }^{-}=\max \left\{\varepsilon_{n}{ }^{-}\right\}$, the maximum value of under-estimation. The LP formulation can be expressed as

minimize

$$
\varepsilon_{3}=\varepsilon_{\mathrm{rng}}=\varepsilon_{\max }^{+}+\varepsilon_{\max }^{-}
$$

subject to

$$
\begin{aligned}
& \sum_{m=1}^{n \leq M} P_{n} U_{n-m+1}-\varepsilon_{\max }^{+} \leq Q_{n}, \quad n=1,2, \ldots, N, \\
& \sum_{m=1}^{n \leq M} P_{n} U_{n-m+1}+\varepsilon_{\max }^{-} \geq Q_{n}, \quad n=1,2, \ldots, N, \\
& \Delta t \sum_{r=1}^{N-M+1} U_{r}=1, \\
& U_{r} \geq 0, \quad r=1,2, \ldots, N-M+1 ; \quad \varepsilon_{\max }^{+} \geq 0, \quad \varepsilon_{\max }^{-} \geq 0
\end{aligned}
$$

This formulation of minimizing the range of errors is somewhat similar to the MLAD formulation which minimizes the largest absolute deviation. However, since the MRNG formulation controls the range of the deviations, it is expected that the UH by the MRNG criterion will yield a better overall fitting and prediction of $\mathrm{DRH}$ than that by the MLAD. The number of constraints for the MRNG formulation is identical to that for the MLAD formulation while the MRNG model has one extra decision variable as compared with the MLAD formulation.

\section{Comparative Studies}

The four LP formulations based upon the criteria of the MSAD, MLAD, MWSAD, and MRNG were applied to derive the UHs based on twenty storm events that occurred in the Nenagh at Clarianna. The drainage area of the watershed is 295 $\mathrm{km}^{2}$. The ERHs and DRHs for the twenty storms were obtained from Bree (1978) and are listed in Table I. The duration of the UH is 3 hours. 
Table I. Data of 20 storms (Bree, 1978) (a) Direct Runoff Hydrographs (DRHs)

\begin{tabular}{|c|c|c|c|c|c|c|c|c|c|c|c|c|c|c|c|c|c|c|c|c|}
\hline \multirow{2}{*}{$\begin{array}{l}\text { Time } \\
\text { (hrs) }\end{array}$} & \multicolumn{20}{|c|}{ DRHs (in cubic meters per second) of Twenty Storms } \\
\hline & 1 & 2 & 3 & 4 & 5 & 6 & 7 & 8 & 9 & 10 & 11 & 12 & 13 & 14 & 15 & 16 & 17 & 18 & 19 & 20 \\
\hline 3 & 2.2 & 0.5 & 1.2 & 1.1 & 0.50 & 0.6 & 0.8 & 2.3 & 1.60 & 1.5 & 1.6 & 2.9 & 1.90 & 1.40 & 1.1 & 0.70 & 1.0 & 1.40 & 0.80 & 1.00 \\
\hline 6 & 5.8 & 1.1 & 2.3 & 2.7 & 1.15 & 1.2 & 1.5 & 2.6 & 3.40 & 3.7 & 11.5 & 7.0 & 6.23 & 4.19 & 2.0 & 1.69 & 8.7 & 1.78 & 1.99 & 2.32 \\
\hline 9 & 11.9 & 3.6 & 3.3 & 7.9 & 2.39 & 4.8 & 4.1 & 6.7 & 11.59 & 9.9 & 18.1 & 14.6 & 15.16 & 12.47 & 3.3 & 4.08 & 18.6 & 2.57 & 4.38 & 6.14 \\
\hline 12 & 15.2 & 7.8 & 5.8 & 13.6 & 9.84 & 18.9 & 5.7 & 10.6 & 15.39 & 12.5 & 13.6 & 15.7 & 22.59 & 18.26 & 7.6 & 4.57 & 16.1 & 25.75 & 7.38 & 20.26 \\
\hline 15 & 15.9 & 10.9 & 11.8 & 13.2 & 13.19 & 16.9 & 9.1 & 11.6 & 12.88 & 13.0 & 9.4 & 29.3 & 25.32 & 20.84 & 12.8 & 5.46 & 12.0 & 35.84 & 22.27 & 30.18 \\
\hline 18 & 12.7 & 12.8 & 15.3 & 27.9 & 8.53 & 11.4 & 14.5 & 11.7 & 8.88 & 10.9 & 7.9 & 41.6 & 20.65 & 17.53 & 13.2 & 9.35 & 8.3 & 38.12 & 26.86 & 32.20 \\
\hline 21 & 10.5 & 12.4 & 13.5 & 17.4 & 7.68 & 8.0 & 12.9 & 12.5 & 6.68 & 8.4 & 6.8 & 40.2 & 13.78 & 12.21 & 10.3 & 9.44 & 7.5 & 24.31 & 23.35 & 32.52 \\
\hline 24 & 10.2 & 9.8 & 10.9 & 15.7 & 7.23 & 7.9 & 10.2 & 13.8 & 5.37 & 6.5 & 6.0 & 28.0 & 10.72 & 9.20 & 7.9 & 6.93 & 5.6 & 18.19 & 16.95 & 23.14 \\
\hline 27 & 10.9 & 8.5 & 8.2 & 18.8 & 5.97 & 7.2 & 9.1 & 12.5 & 4.57 & 5.5 & 5.3 & 21.7 & 8.85 & 8.38 & 7.0 & 5.42 & 5.2 & 16.18 & 13.84 & 16.26 \\
\hline 30 & 12.3 & 7.3 & 6.8 & 17.9 & 4.82 & 6.3 & 8.0 & 11.2 & 3.77 & 4.6 & 4.7 & 20.5 & 6.98 & 6.17 & 6.7 & 4.71 & 4.7 & 14.66 & 11.43 & 12.58 \\
\hline 33 & 11.2 & 6.2 & 5.9 & 15.3 & 4.07 & 5.2 & 7.3 & 10.3 & 2.96 & 3.9 & 4.3 & 22.9 & 6.21 & 5.35 & 6.7 & 4.00 & 4.3 & 13.65 & 8.92 & 10.10 \\
\hline 36 & 9.6 & 5.3 & 5.2 & 12.8 & 3.61 & 4.4 & 6.8 & 9.7 & 2.46 & 3.4 & 3.8 & 21.7 & 5.44 & 4.54 & 6.0 & 3.29 & 3.9 & 12.23 & 7.42 & 9.22 \\
\hline 39 & 9.0 & 4.6 & 4.8 & 11.2 & 3.26 & 3.9 & 6.8 & 8.8 & 1.95 & 2.9 & 3.3 & 18.7 & 4.67 & 3.92 & 5.5 & 2.98 & 3.5 & 10.92 & 6.61 & 7.34 \\
\hline 42 & 8.4 & 3.8 & 4.3 & 9.7 & 3.11 & 3.3 & 6.3 & 7.1 & 1.65 & 2.0 & 3.1 & 16.9 & 4.80 & 3.41 & 4.9 & 2.67 & 3.2 & 11.20 & 5.70 & 6.56 \\
\hline 45 & 8.0 & 3.1 & 4.0 & 9.1 & 2.95 & 3.1 & 5.8 & 6.3 & 1.55 & 2.2 & 2.8 & 12.3 & 4.23 & 3.09 & 4.4 & 2.46 & 3.0 & 10.28 & 4.99 & 5.68 \\
\hline
\end{tabular}




\begin{tabular}{|c|c|c|c|c|c|c|c|c|c|c|c|c|c|c|c|c|c|c|c|c|}
\hline 48 & 7.4 & 2.4 & 3.5 & 8.5 & 2.80 & 2.9 & 5.3 & 5.5 & 1.24 & 1.8 & 2.4 & 10.7 & 3.46 & 2.58 & 3.9 & 2.24 & 2.8 & 10.07 & 4.28 & 4.90 \\
\hline 51 & 6.7 & 2.1 & 3.1 & 7.9 & 2.75 & 2.7 & 4.9 & 4.8 & 1.04 & 1.6 & 2.1 & 9.6 & 3.29 & 2.56 & 3.0 & 2.13 & 2.6 & 9.15 & 3.38 & 4.12 \\
\hline 54 & 6.1 & 1.6 & 2.7 & 7.1 & 2.40 & 2.2 & 4.4 & 4.1 & 0.93 & 1.3 & 1.8 & 7.5 & 3.02 & 2.15 & 2.5 & 1.92 & 2.4 & 8.94 & 2.67 & 3.34 \\
\hline 57 & 5.5 & 1.2 & 2.3 & 6.4 & 2.14 & 1.8 & 4.0 & 3.5 & 0.83 & 1.0 & 1.5 & 5.9 & 2.75 & 1.83 & 2.1 & 1.61 & 2.2 & 8.02 & 2.36 & 2.56 \\
\hline 60 & 5.1 & 1.0 & 2.0 & 5.9 & 2.14 & 1.5 & 3.5 & 3.0 & 0.73 & 0.8 & 1.2 & 4.9 & 2.38 & 1.62 & 1.8 & 1.50 & 2.0 & 7.31 & 2.05 & 1.38 \\
\hline 63 & 4.6 & 0.9 & 1.8 & 5.3 & 1.94 & 1.2 & 3.1 & 2.5 & 0.62 & 0.7 & 1.0 & 3.5 & 2.02 & 1.40 & 1.5 & 1.19 & 1.8 & 6.79 & 1.75 & 1.00 \\
\hline 66 & 4.1 & 0.6 & 1.7 & 4.7 & 1.78 & 1.0 & 2.7 & 2.2 & 0.52 & 0.6 & 0.9 & 2.7 & 1.65 & 1.19 & 1.2 & 0.98 & 1.6 & 6.68 & 1.44 & 0.72 \\
\hline 69 & 3.6 & 0.5 & 1.5 & 4.3 & 1.63 & 0.8 & 2.3 & 1.9 & 0.42 & 0.5 & 0.7 & 2.2 & 1.28 & 0.97 & 0.9 & 0.57 & 1.4 & 6.16 & 1.13 & 0.54 \\
\hline 72 & 3.1 & 0.4 & 1.3 & 3.8 & 1.38 & 0.6 & 1.9 & 1.6 & 0.31 & 0.4 & 0.6 & 1.8 & 0.91 & 0.76 & 0.7 & 0.56 & 1.2 & 5.65 & 0.82 & 0.36 \\
\hline 75 & 2.6 & 0.3 & 1.1 & 3.5 & 1.12 & 0.4 & 1.5 & 1.3 & 0.21 & 0.3 & 0.5 & 1.5 & 0.54 & 0.54 & 0.5 & 0.25 & 1.0 & 5.03 & 0.52 & 0.18 \\
\hline 78 & 2.1 & 0.2 & 1.0 & 3.1 & 0.97 & 0.2 & 1.2 & 1.2 & 0.10 & 0.2 & 0.4 & 1.3 & 0.27 & 0.43 & 0.3 & 0.04 & 0.8 & 4.42 & 0.21 & \\
\hline 81 & 1.7 & & 0.8 & 2.6 & 0.82 & & 0.9 & 1.1 & & 0.1 & 0.3 & 1.1 & & 0.21 & 0.1 & 0.03 & 0.6 & 3.70 & & \\
\hline 84 & 1.3 & & 0.7 & 2.2 & 0.66 & & 0.6 & 0.9 & & & 0.2 & 0.9 & & & & 0.02 & 0.4 & 3.28 & & \\
\hline 87 & 0.9 & & 0.5 & 1.8 & 0.41 & & 0.3 & 0.8 & & & 0.1 & 0.7 & & & & & 0.2 & 2.77 & & \\
\hline 90 & 0.6 & & 0.4 & 1.4 & 0.26 & & 0.1 & 0.7 & & & & 0.6 & & & & & 0.1 & 2.55 & & \\
\hline 93 & 0.3 & & 0.2 & 1.1 & 0.10 & & & 0.5 & & & & 0.5 & & & & & & 1.94 & & \\
\hline 96 & & & 0.1 & 0.8 & & & & 0.3 & & & & 0.4 & & & & & & 1.72 & & \\
\hline 99 & & & & 0.5 & & & & 0.2 & & & & 0.3 & & & & & & 1.51 & & \\
\hline 102 & & & & & & & & 0.1 & & & & 0.2 & & & & & & 1.29 & & \\
\hline 105 & & & & & & & & & & & & 0.1 & & & & & & 1.08 & & \\
\hline
\end{tabular}


Table I. Data of 20 storms (Bree, 1978)

(b) Effective rainfall hyetographs (ERHs)

\begin{tabular}{|c|c|c|c|c|c|c|c|c|c|c|c|c|c|c|c|c|c|c|c|c|}
\hline \multirow{2}{*}{$\begin{array}{l}\text { Time } \\
\text { (hrs) }\end{array}$} & \multicolumn{20}{|c|}{ ERH (mm) of Twenty Storms } \\
\hline & 1 & 2 & 3 & 4 & 5 & 6 & 7 & 8 & 9 & 10 & 11 & 12 & 13 & 14 & 15 & 16 & 17 & 18 & 19 & 20 \\
\hline 3 & 1.57 & 0.48 & 0.76 & 2.17 & 0.22 & 1.42 & 1.69 & 0.70 & 0.62 & 0.87 & 2.19 & 1.25 & 2.85 & 1.74 & 0.76 & 0.59 & 1.09 & 1.74 & 1.20 & 5.64 \\
\hline 6 & 1.92 & 0.58 & 0.57 & 2.12 & 0.44 & 2.16 & 0.92 & 1.11 & 1.88 & 1.98 & 0.81 & 2.97 & 2.32 & 2.04 & 0.42 & 0.96 & 1.82 & 3.66 & 1.79 & 2.94 \\
\hline 9 & 2.39 & 2.92 & 0.54 & 0.92 & 1.44 & 0.75 & 1.11 & 1.59 & 0.85 & 0.36 & 0.49 & 0.10 & 1.38 & 1.41 & 2.67 & 0.03 & 0.34 & 0.75 & 2.73 & \\
\hline 12 & 0 & & 0.81 & 0.22 & 0.06 & & 1.13 & 0.54 & & 0.45 & 0.32 & 2.42 & & 0.20 & 0.46 & 0.75 & 0.33 & 0.72 & 0.99 & \\
\hline 15 & 0 & & 1.02 & 0.47 & 0.29 & & 0.19 & 0.48 & & & 0.29 & 3.06 & & & & 0.64 & 0.33 & 0.28 & & \\
\hline 18 & 0 & & 0.25 & 0.97 & 0.58 & & 0.18 & 0.34 & & & 0.15 & 0.23 & & & & & 0.33 & 1.22 & & \\
\hline 21 & 0.14 & & 0.25 & 0.84 & 0.38 & & 0.1 & 0.55 & & & & 0.83 & & & & & 0.33 & 0.09 & & \\
\hline 24 & 1.64 & & 0.25 & 0.80 & 0.32 & & & 0.51 & & & & 0.07 & & & & & & 0.33 & & \\
\hline 27 & & & 0.25 & 0.71 & & & & 0.06 & & & & 0.20 & & & & & & 0.67 & & \\
\hline 30 & & & & 0.49 & & & & 0.32 & & & & 1.60 & & & & & & 0.43 & & \\
\hline 33 & & & & & & & & 0.16 & & & & 0.43 & & & & & & 0.21 & & \\
\hline 36 & & & & & & & & & & & & 0.39 & & & & & & 0.30 & & \\
\hline 39 & & & & & & & & & & & & & & & & & & 0.41 & & \\
\hline 42 & & & & & & & & & & & & & & & & & & 0.57 & & \\
\hline 45 & & & & & & & & & & & & & & & & & & 0.59 & & \\
\hline 48 & & & & & & & & & & & & & & & & & & 0.35 & & \\
\hline
\end{tabular}


Computer programs using MATLAB language were developed to conduct the analysis. MATLAB language is a high-level computer language which is efficient in matrix operations (Mathworks, 1989). The LP algorithm in MATLAB is based upon an active set method which is a variation of the simplex method (Grace, 1993). All computer programs written in MATLAB were run on a PC-486DX with $33 \mathrm{MHz}$.

\subsection{REGENERATION AND PREDICTION OF DRHS BY THE DERIVED UH}

An optimal UH can be obtained by solving one of the four LP formulations based upon the ERH and DRH of a storm. Regeneration of a DRH is referred to as using the obtained UH to compute or regenerate the DRH for the storm used in the derivation of the optimal UH. Equation (1) can be used to convolve the derived optimal UH with the ERH to regenerate the corresponding DRH. On the other hand, prediction of a DRH herein is referred to as using the derived UH to compute the DRH of another storm by convolving the derived optimal UH with the ERH data of the corresponding storm.

A UH obtained from minimizing one form of error criterion is optimal only to the error criterion describing the deviation between the regenerated and the observed DRH that is minimized. The error between the regenerated and observed DRHs is minimal for the error criterion used in derived the optimal UH. However, the optimality for a regenerated storm does not necessarily translate to the optimality for predicting another storm. Therefore, it is important to investigate how well derived UH can predict the DRHs for other storms. A comparison of predictability among the optimal UHs obtained from the MSAD, MLAD, MWSAD, and MRNG will be presented later. A comparison based upon a statistical cross-validation technique is given below.

\subsection{STATISTICAL CROSS-VALIDATION TECHNIQUE}

Validation is an important task in the process of developing a model. Based upon validation test, one can assess how well the derived UH can predict the DRHs of future storms. The statistical cross-validation method is a useful technique (Allen, 1971; Stone, 1974; Geisser, 1975; McCarthy, 1976). When applied to a UH validation test where $R$ storms are available, the cross-validation method uses one of the $R$ storms to obtain an optimal $\mathrm{UH}$ and then uses the optimal $\mathrm{UH}$ to predict the DRHs for the remaining $R-1$ storms. The errors between the observed and predicted DRHs for each storm can be computed by the adopted error criterion from which an average error over the remaining $R$ storms can be computed. This process is repeated for each of the $R$ storms. Then, a final average error value can be computed which is used to compare the predictability of UHs obtained from different LP formulations. The procedure of the statistical cross-validation methods 
if as follows:

(1) Consider $R$ storms, $S_{1}, S_{2}, \ldots, S_{R}$.

(2) Let $r=1$.

(3) Apply a LP formulation (MSAD, MLAD, MWSAD, or MRNG) to storm $S_{r}$ to obtain an optimal $\mathrm{UH}_{r}$.

(4) Use the optimal $\mathrm{UH}_{r}$ to predict the DRHs of the remaining $R-1$ storms.

(5) Compute the error measure of interest, $E_{r}$, based on the deviations between the predicted and observed DRH for each of the $R-1$ storms.

(6) Average $E_{r}$ over the $R-1$ storms to obtain $\bar{E}_{r}$.

(7) Repeat steps (3)-(6) for $r=r+1$ until $r=R$.

(8) Average $\bar{E}_{r}$ over $R$ storms to obtain an averaged error measure to be used for comparing the predictability of UH from different LP formulations.

\subsection{ERROR CRITERIA}

Eleven error criteria were considered in this study for assessing the predictability and reproducibility of DRHs by the UHs from the four LP formulations. They are

(1) The sum of absolute deviations of DRH ordinates:

$$
E_{r, 1}=\sum_{n=1}^{N_{r}}\left|\hat{\mathrm{Q}}_{r, n}-Q_{r, n}\right|
$$

in which $Q_{r, n}$ is the observed $n$th DRH ordinate for the $r$ th storm and the " $\wedge$ " represents the regenerated or predicted values, and $N_{r}$ is the number of DRH ordinates for the $r$ th storm.

(2) The weighted sum of absolute deviations of DRH ordinates:

$$
E_{r, 2}=\sum_{n=1}^{N_{r}} W_{n}\left|\hat{Q}_{r, n}-Q_{r, n}\right|,
$$

where the weighing function $W_{n}$ is defined by Equation (5).

(3) The largest absolute deviation of DRH ordinates:

$$
E_{r, 3}=\max _{1 \leq n \leq N}\left|\hat{Q}_{r, n}-Q_{r, n}\right| \text {. }
$$

(4) The range of deviations of DRH ordinates:

$$
E_{r, 4}=\max _{1 \leq n \leq N}\left(\hat{\mathrm{Q}}_{r, n}-Q_{r, n}\right)+\max _{1 \leq n \leq N} Q_{r, n}-\left(\hat{\mathrm{Q}}_{r, n}\right) .
$$

(5) The root-mean-squared error of DRH ordinates:

$$
E_{r, 5}=\sqrt{\frac{\sum_{n=1}^{N_{r}}\left(\hat{Q}_{r, n}-Q_{r, n}\right)^{2}}{N_{r}}} .
$$


(6) The relative absolute error of time-to-peak:

$$
E_{r, 6}=\left|\frac{\widehat{T P}_{r}-T P_{r}}{T P_{r}}\right|
$$

in which $\mathrm{TP}_{r}$ is the time-to-peak of DRH of the $r$ th storm.

(7) The relative absolute error of $\mathrm{DRH}$ peak:

$$
E_{r, 7}=\left|\frac{\widehat{Q P}_{r}-Q P_{r}}{Q P_{r}}\right|
$$

in which $\mathrm{QP}_{r}$ is the peak discharge of $\mathrm{DRH}$ of the $r$ th storm.

(8) The relative absolute error of DRH volume:

$$
E_{r, 8}=\left|\frac{\hat{V}_{r}-V_{r}}{V_{r}}\right|
$$

in which $V_{r}$ is the volume of DRH of the $r$ th storm.

(9) The relative bias of time-to-peak:

$$
E_{r, 9}=\frac{\widehat{T P}_{r}-T P_{r}}{T P_{r}} .
$$

(10) The relative bias of DRH peak:

$$
E_{r, 10}=\frac{\widehat{Q P}_{r}-Q P_{r}}{Q P_{r}} .
$$

(11) The relative bias of DRH volume:

$$
E_{r, 11}=\frac{\hat{V}_{r}-V_{r}}{V_{r}}
$$

Error criteria (1)-(4) coincide with the objective functions of the MSAD, MLAD, MWSAD, and MRNG, respectively. Also, note that error criteria (9)(11) are useful to measure the over-prediction or under-prediction of time-to-peak, peak, and volume of DRH. Suppose that the prediction of a DRH is considered. A positive $E_{9}$ implies an overall over-prediction of time-to-peak in the predicted DRH whereas a negative $E_{9}$ implies an overall under-prediction of the time-to-peak.

\subsection{COMPARISON OF THE DERIVED OPTIMAL UHS}

An optimal UH can be obtained from each of the 20 storms by solving each of the LP formulations. The LP formulations are the MSAD, MLAD, MWSAD with $\alpha=-0.5$, MWSAD with $\alpha=0.5$, and MRNG. Figure 1 illustrates the UHs for one of the 20 storms by different LP formulations. The comparison among the optimal UHs obtained from the five LP formulations was made based upon the averaged value for a UH considered herin property over the 20 storms. The properties of a UH are the peak, time-to-peak, smoothness, computation time, number of decision 


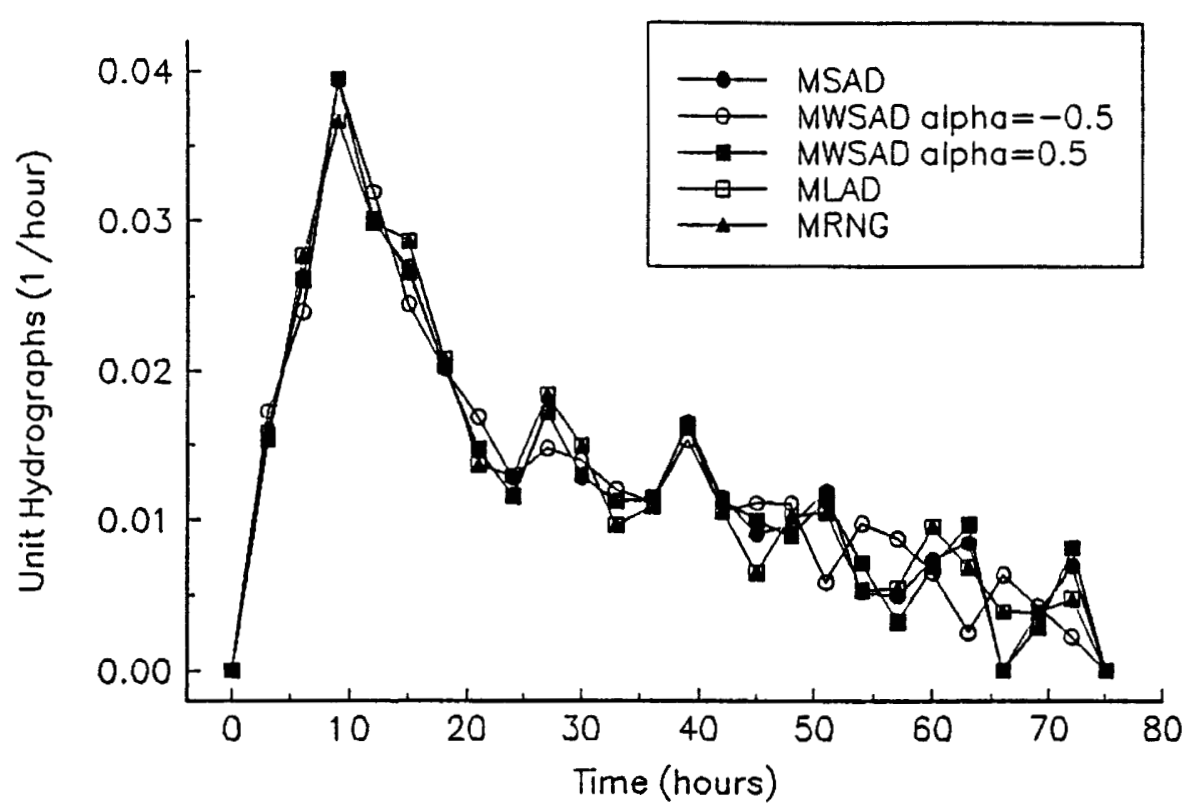

Fig. 1. UHs for storm No. 1 by different LP formulations.

variables, and number of constraints. Smoothness of a UH, based upon Bkörck (1990), can be measured by

$$
\|\mathbf{B u}\|_{2}
$$

where $\mathbf{u}$ is a vector of derived $\mathrm{UH}$ ordinates and $\mathbf{B}$ is a matrix of discrete approximation to the second-derivative operator,

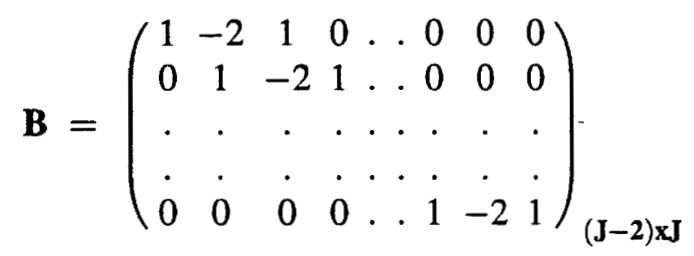

Table II lists the averaged values for each of the UH properties over the twenty storms. It can be seen in Table II that the MLAD formulation requires the least computation time while the MSAD and MWSAD models require the most computation time. The computation time for the MRNG formulation is between the time required by the MLAD and MSAD (or MWSAD). Recall that, with $N$ and $M$ respectively being the number of ordinates of a DRH and ERH, the number of decision variables for the models of MSAD (or MWSAD), MLAD, and MRNG are $3 \mathrm{~N}$ $\mathrm{M}+1, \mathrm{~N}-\mathrm{M}+2$, and $\mathrm{N}-\mathrm{M}+3$, respectively. The number of constraints in the models of MSAD (or MWSAD) and MLAD (or MRNG) are $N+1$ and $2 \mathrm{~N}+1$, respectively. The numerical results show that the averaged computation time increases as 
TABLE II. Averaged values associated with UHs based upon 20 storms for different formulations

\begin{tabular}{llllll}
\hline UH Properties & MSAD & $\begin{array}{l}\text { MWSAD } \\
(\alpha=-0.5)\end{array}$ & $\begin{array}{l}\text { MWSAD } \\
(\alpha=0.5)\end{array}$ & MLAD & MRNG \\
\hline Computing time (sec) & $7.0639 \mathrm{e}+01$ & $7.319 \mathrm{e}+01$ & $7.3440 \mathrm{e}+01$ & $1.3626 \mathrm{e}+01$ & $2.9744 \mathrm{e}+01$ \\
Number of variables & 83 & 83 & 83 & 25 & 26 \\
Number of Contraints & 30 & 30 & 30 & 60 & 60 \\
Peak (1/hr) & $6.0994 \mathrm{e}-02$ & $6.1472 \mathrm{e}-02$ & $6.0169 \mathrm{e}-02$ & $6.2281 \mathrm{e}-02$ & $6.2281 \mathrm{e}-02$ \\
Time-to-Peak (hours) & $1.2150 \mathrm{e}+01$ & $1.2150 \mathrm{e}+01$ & $1.1700 \mathrm{e}+01$ & $1.2300 \mathrm{e}+01$ & $1.2300 \mathrm{e}+01$ \\
Smoothness & $1.1297 \mathrm{e}-02$ & $8.0704 \mathrm{e}-03$ & $9.5429 \mathrm{e}-03$ & $1.4039 \mathrm{e}-02$ & $1.3831 \mathrm{e}-02$ \\
Volume & 1.0 & 1.0 & 1.0 & 1.0 & 1.0 \\
\hline
\end{tabular}

TABLE III. Averaged values of error criteria associated with regenerated DRHs based upon 20 storms for different formulations

\begin{tabular}{lrrrr}
\hline $\begin{array}{l}\text { Error criteria for } \\
\text { regenerated DRHs } \\
\text { properties }\end{array}$ & MSAD & \multicolumn{1}{l}{ MWSAD } \\
$\alpha=0.5$ & \multicolumn{1}{l}{ MLAD } & MRNG \\
\hline $\mathrm{E}_{r, 1}$ (MSAD) & $5.9338 \mathrm{e}+00$ & $6.5864 \mathrm{e}+00$ & $1.7581 \mathrm{e}+01$ & $1.7030 \mathrm{e}+01$ \\
$\mathrm{E}_{r, 2}$ (MWSAD, $\left.\alpha=0.5\right)$ & $7.0448 \mathrm{e}+00$ & $6.2350 \mathrm{e}+00$ & $2.0619 \mathrm{e}+01$ & $2.0147 \mathrm{e}+01$ \\
$\mathrm{E}_{r, 3}$ (MLAD) & $1.8137 \mathrm{e}+00$ & $2.0818 \mathrm{e}+00$ & $8.4104 \mathrm{e}-01$ & $8.4104 \mathrm{e}-01$ \\
$\mathrm{E}_{r, 4}$ (MRNG) & $2.8573 \mathrm{e}+00$ & $3.0489 \mathrm{e}+00$ & $1.6820 \mathrm{e}+00$ & $1.6820 \mathrm{e}+00$ \\
$\mathrm{E}_{r, 5}$ (RMSE) & $4.4787 \mathrm{e}-01$ & $4.8899 \mathrm{e}-01$ & $6.3673 \mathrm{e}-01$ & $6.2505 \mathrm{e}-01$ \\
$\mathrm{E}_{r, 6}$ (Time-to-peak) & $2.7976 \mathrm{e}-02$ & $8.3333 \mathrm{e}-03$ & $1.8333 \mathrm{e}-02$ & $1.8333 \mathrm{e}-02$ \\
$\mathrm{E}_{r, 7}$ (Peak) & $2.7580 \mathrm{e}-02$ & $1.8771 \mathrm{e}-02$ & $3.3626 \mathrm{e}-02$ & $3.3763 \mathrm{e}-02$ \\
$\mathrm{E}_{r, 8}$ (Volume) & $1.4924 \mathrm{e}-03$ & $1.4924 \mathrm{e}-03$ & $1.4924 \mathrm{e}-03$ & $1.4924 \mathrm{e}-03$ \\
$\mathrm{E}_{r, 9}$ (Time-to-peak) & $1.3690 \mathrm{e}-02$ & $8.3333 \mathrm{e}-03$ & $-1.8333 \mathrm{e}-02$ & $-1.8333 \mathrm{e}-02$ \\
$\mathrm{E}_{r, 10}$ (Peak) & $-2.7580 \mathrm{e}-02$ & $-1.8771 \mathrm{e}-02$ & $-1.1894 \mathrm{e}-02$ & $-1.1757 \mathrm{e}-02$ \\
$\mathrm{E}_{r, 11}$ (Volume) & $-1.8833 \mathrm{e}-04$ & $-1.8833 \mathrm{e}-04$ & $-1.8833 \mathrm{e}-04$ & $-1.8833 \mathrm{e}-04$ \\
\hline
\end{tabular}

Note: The units for MSAD, MWSAD, MLAD, MRNG, and RMSE are $\mathrm{m}^{3} / \mathrm{s}$.

the averaged number of decision variables increases by the active set algorithm employed in MATLAB.

One can observe from Table II that the UHs obtained from the MLAD and MRNG formulations have the same peak and time-to-peak which are larger than those from the MSAD and MWSAD. The smoothness values in Table II indicate that the UH obtained from the MRNG formulation is smoother than that obtained from the MLAD. The UH from the MWSAD formulation with $\alpha=-0.5$ is the smoothest. The volume of all derived UHs is unity as expected.

\subsection{COMPARISON OF THE REPRODUCED DRHS}

The optimal UH obtained from solving LP models of MSAD, MWSAD with $\alpha=0.5$, MLAD, and MRNG to each of the 20 storms is used to regenerate the 
DRH of the storm used to derive the optimal UH. The error measures between the reproduced and observed DRHs are measured by the eleven criteria described in Section 4.3. Then, the averaged values for each of the error criteria is computed over the 20 storms. It can be observed in Table III that the UHs by MSAD, MWSAD with $\alpha=0.5, \mathrm{MLAD}$, and MRNG regenerate DRHs which have, on an average, the smalles values of $E_{r, 1}, E_{r, 2}, E_{r, 3}$, and $E_{r, 4}$, respectively. This is expected because the error criteria coincide with the objective functions which are minimized. It is also shown in Table III that the UH from the MRNG yields a smaller value of $E_{r, 1}$ than the MLAD does. This implies that the UH from the MRNG is better in overall curve-fitting than that from the MLAD.

Other error criteria such as the RMSE, time-to-peak, peak, and volume defined in Section 4.3 were also computed and tabulated in Table III. As expected, the MSAD yields the smallest value of the RMSE. Comparing the time-to-peak error $\left(E_{r, 6}\right)$ among different LP formulations, one can find that the MWSAD with $\alpha=0.5$ gives the smallest value of $E_{r, 6}$ and the MSAD gives the largest value. As for the peak discharge error $\left(E_{r, 7}\right.$, the MWSAD with $\alpha=0.5$ results in the smallest value. The volume errors $\left(E_{r, 8}\right)$ for the different LP formulations are the same.

Criteria $E_{r, 9}, E_{r, 10}$, and $E_{r, 11}$ were used to evaluate the degrees of bias of the reproduced DRH with respect to the time-to-peak, peak, or volume compared with the observed DRH. This bias criterion may be important in flood forecasting. Since the values of $E_{r, 9}$ for the MLAD and MRNG are negative (see Table III), the time-to-peak of the regenerated DRHs by the MLAD and MRNG is shorter, on an average, than that of the observed DRHs. The negative values for peak and volume errors of the regenerated DRHs for all LP formulations indicate that all LP formulations result in under-estimating the peak and volume of DRHs.

\subsection{COMPARISON OF PREDICTED DRHS}

An important application of the UH theory is to use the derived UH to predict the DRHs of future storms. Therefore, it is essential to study the predictability of the derived UH by the various LP formations.

The algorithm of the statistical cross-validation described in Section 4.2 was applied to the 20 storms in Bree (1978). $E_{1}$ and $E_{5}$ were used to measure the overall prediction of DRHs. Table IV shows that the MSWAD with $\alpha=-0.5$ yields the smallest values of $E_{1}$ and $E_{5}$. This indicates that the MSWAD with $\alpha=-0.5$ is the best in overall prediction of DRHs for other storms or possibly future storms. Examining the eleven error criteria $\left(E_{1}-E_{11}\right)$ for all LP formulations, one observes that the MWSAD with $\alpha=-0.5$ gives the smallest error values of $E_{1}-E_{11}$ than other LP formulations do, implying that the MWSAD with $\alpha=-0.5$ has the best predictability. When a negative value is assigned to $\alpha$ in Equation (5), higher discharges in the DRH receive less weight than those lower discharges. The results having a better predictability with a negative-valued $\alpha$ indicate that, contrary to the popular belief in common hydrologic practice, emphasis to fit high discharge in 
TABLE IV. Averaged values of error criteria associated with predicted DRHs based upon statistical cross-validation on 20 storms for different LP formulations

\begin{tabular}{lrlllr}
\hline $\begin{array}{l}\text { Error criteria for } \\
\text { regenerated DRHs } \\
\text { properties }\end{array}$ & MSAD & $\begin{array}{l}\text { MWSAD } \\
\alpha=-0.5\end{array}$ & \multicolumn{1}{l}{$\begin{array}{l}\text { MWSAD } \\
(\alpha=0.5)\end{array}$} & MLAD & MRNG \\
\hline $\mathrm{E}_{1}$ (MSAD) & $6.0502 \mathrm{e}+01$ & $5.8462 \mathrm{e}+01$ & $6.1361 \mathrm{e}+01$ & $6.2433 \mathrm{e}+01$ & $6.2397 \mathrm{e}+01$ \\
$\mathrm{E}_{2}$ (MWSAD, $\left.\alpha=0.5\right)$ & $8.3742 \mathrm{e}+01$ & $3.6706 \mathrm{e}+01 *$ & $8.4702 \mathrm{e}+01$ & $8.5878 \mathrm{e}+01$ & $8.5907 \mathrm{e}+01$ \\
$\mathrm{E}_{3}$ (MLAD) & $9.8833 \mathrm{e}+00$ & $9.7240 \mathrm{e}+00$ & $9.8574 \mathrm{e}+00$ & $9.9342 \mathrm{e}+00$ & $9.9342 \mathrm{e}+00$ \\
$\mathrm{E}_{4}$ (MRNG) & $1.5718 \mathrm{e}+01$ & $1.5377 \mathrm{e}+01$ & $1.5789 \mathrm{e}+01$ & $1.6112 \mathrm{e}+01$ & $1.6093 \mathrm{e}+01$ \\
$\mathrm{E}_{5}$ (RMSE) & $3.1511 \mathrm{e}+00$ & $3.0655 \mathrm{e}+00$ & $3.1822 \mathrm{e}+00$ & $3.2258 \mathrm{e}+00$ & $3.2238 \mathrm{e}+00$ \\
$\mathrm{E}_{6}$ (Time-to-peak) & $2.7080 \mathrm{e}-01$ & $2.5844 \mathrm{e}-01$ & $2.7386 \mathrm{e}-01$ & $2.7001 \mathrm{e}-01$ & $2.7001 \mathrm{e}-01$ \\
$\mathrm{E}_{7}$ (Peak) & $2.2970 \mathrm{e}-01$ & $2.2704 \mathrm{e}-01$ & $2.3292 \mathrm{e}-01$ & $2.2757 \mathrm{e}-01$ & $2.2759 \mathrm{e}-01$ \\
$\mathrm{E}_{8}$ (Volume) & $1.4928 \mathrm{e}-03$ & $1.4928 \mathrm{e}-03$ & $1.4928 \mathrm{e}-03$ & $1.4928 \mathrm{e}-03$ & $1.4928 \mathrm{e}-03$ \\
$\mathrm{E}_{9}$ (Time-to-peak) & $8.3377 \mathrm{e}-02$ & $7.0617 \mathrm{e}-02$ & $7.6901 \mathrm{e}-02$ & $9.9479 \mathrm{e}-02$ & $9.9479 \mathrm{e}-02$ \\
$\mathrm{E}_{10}$ (Peak) & $1.5303 \mathrm{e}-02$ & $6.7154 \mathrm{e}-03$ & $2.0161 \mathrm{e}-02$ & $2.4634 \mathrm{e}-02$ & $2.4649 \mathrm{e}-02$ \\
$\mathrm{E}_{11}$ (Volume) & $-1.3859 \mathrm{e}-04$ & $-1.3859 \mathrm{e}-04$ & $-1.3859 \mathrm{e}-04$ & $-1.3859 \mathrm{e}-04$ & $-1.3859 \mathrm{e}-04$ \\
\hline
\end{tabular}

Notes:

1 The units for MSAD, MWSAD, MLAD, MRNG, and RMSE are $\mathrm{m}^{3} / \mathrm{s}$.

2 Supscript '*' indicates that the error criterion $E_{r, 2}$ uses $\alpha=-0.5$ instead of 0.5 .

UH determination does not necessarily yield accuracy prediction for future storm events as a whole or even for the peak discharge of future events. Criterion $E_{7}$ in Table IV shows that the overall accuracy of predicting peak discharge of DRH for other storms by the MLAD and MRNG is better than the MSAD and MWSAD with $\alpha=0.5$. Criterion $E_{10}$ in Table IV indicates that to emphasize fitting to higher discharges potentially would lead to over-prediction of peak discharge of future storms. If one argues on the viewpoint of conservatism, using the MLAD and MRNG formulations could be even more conservative on prediciting peak discharge.

Table IV also shows that the MRNG yields smaller values of $E_{1}$ and $E_{5}$ than the MLAD does. The MLAD and MRNG have the same error values for time-topeak, peak, and volume for predicting the DRHs. It is interesting to note that the MWSAD with $\alpha=0.5$ results in larger values of $E_{1}$ and $E_{2}$ than the MSAD does but smaller values than the MLAD and MRNG do.

\subsection{DETERMINATION OF OPTIMAL WEIGHTS FOR MWSAD FORMULATION}

The numerical results in Section 4.6 based upon statistical cross-validation show that the MWSAD formulation with a negative $\alpha$ is better than the one with $\alpha \geq 0$ in predicting the DRH. Note that the MWSAD reduces to MSAD when $\alpha=0$. In this section, an optimal $\alpha$ is determined by golden section technique with the embedded statistical cross-validation method such that an adopted error criterion is minimized. The golden section technique is a one-dimensional optimum search 


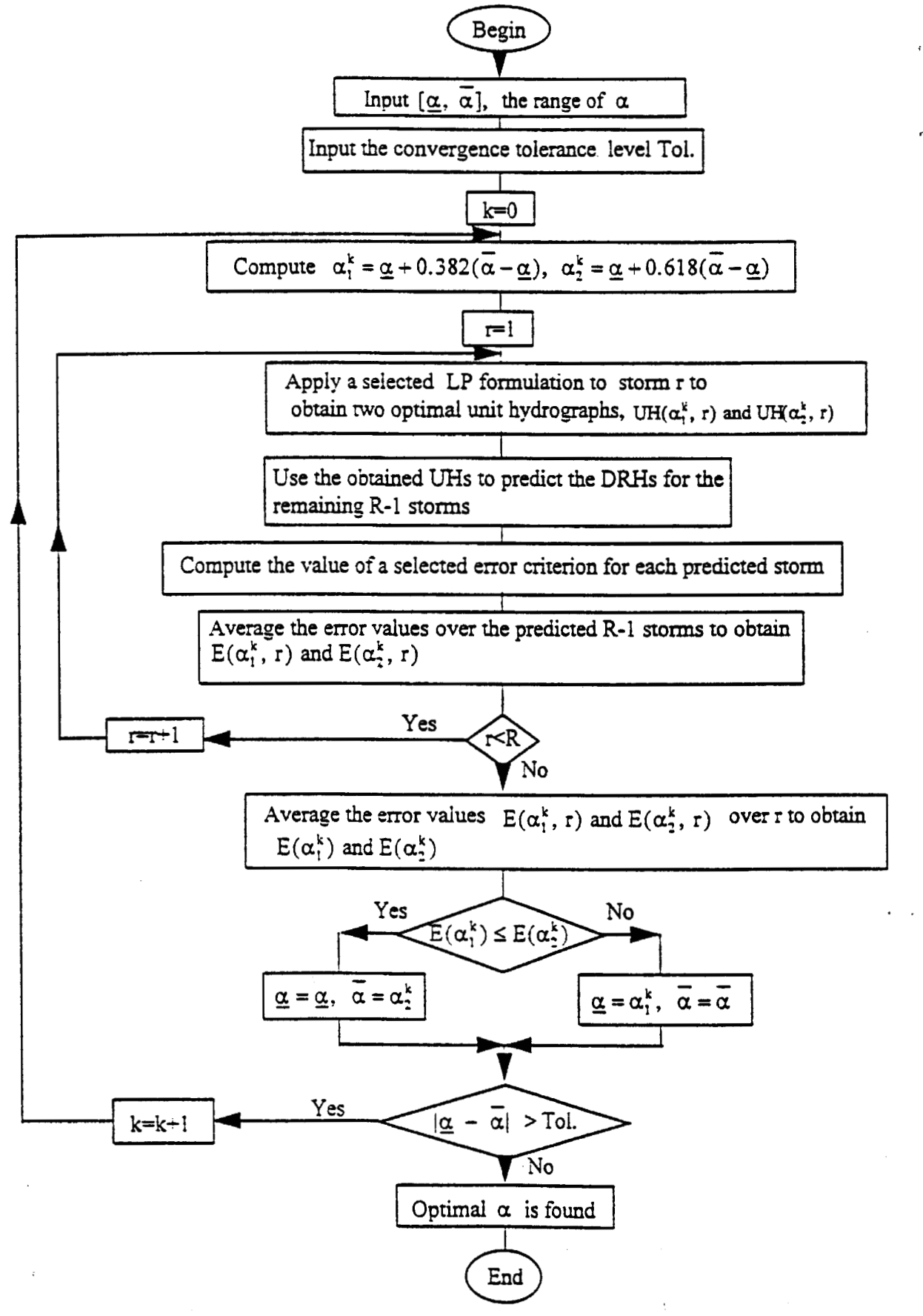

Fig. 2. Flowchart for determining an optimal weight function by the golden section technique in conjunction with the statistical cross-validation method for LP formulation. 
OPTIMAL UNIT HYDROGRAPHS AND LINEAR PROGRAMMINGS

TABLE V. Optimal $\alpha$ by the golden section technique for different prediction error criteria in conjunction with the statistical cross-validation technique

\begin{tabular}{llll}
\hline & Error criteria & \\
\cline { 2 - 4 } & $E_{1}$ (MSAD) & $E_{6}$ (Time-to-Peak) & $E_{7}$ (Peak) \\
\hline Convergance tolerance $\alpha$ & 0.001 & 0.001 & 0.001 \\
Iteration number & 18 & 18 & 18 \\
Computing time & 15 hours & 15 hours & 15 hours \\
Optimal $\alpha$ & -0.68 & -0.33 & -0.68 \\
Minimized mean & $58.384\left(\mathrm{~m}^{3} / \mathrm{s}\right)$ & 0.2235 & 0.00149 \\
\hline
\end{tabular}

procedure. Detailed discussion on the golden section technique can be found in Mays and Tung (1992). Figure 2 gives a flow chart for the determination of the optimal $\alpha$ for the MWSAD model by the golden section technique in conjunction with the statistical-cross validation.

Table $\mathrm{V}$ lists the optimal values of $\alpha$ for three different error criteria, namely, $E_{1}$, $E_{6}$ and $E_{7}$, for the predicted DRHs. By the golden section technique, the optimal $\alpha$ with respect to each of the three error criteria were searched in the interval $(-2$, 2 ). With 20 storms in validation test, each error criterion takes eighteen iterations and about fifteen hours on a PC-486 to converge to an optimal solution.

\section{Summary and Conclusions}

The main purposes of this paper were (1) to propose two alternative LP formulations for determining the optimal UHs and (2) to asssess the relative performance of the derived UHs based upon various LP formulations. The two proposed alternative LP formulation are to minimize the weighted sum of absolue deviations and to minimize the range of deviations. The MWSAD formulation is a generalization of the commonly used MSAD model for UH determination. The MRNG model may also be considered as the extension of minimizing the largest deviation (MLAD). The performance assessment were conducted using a statistical cross-validation technique based on 20 storms from Bree (1978).

The comparison among various LP formulations was made based upon (1) UH properties such as peak, time-to-peak, volume, CPU time for computing an optimal UH, number of decision variables, number of constraints; (2) properties of the regenerated DRHs; and (3) properties of the predicted DRHs. Golden section technique was applied to determine the optimal weighing function for the MWSAD formulation in conjunction with the statistical cross-validation technique.

The numerical results from the statistical cross-validation show that the MRNG gives a smoother UH than the MDLAD does. The regenerated DRHs and the predicted DRHs by the MRNG are closer to the observed DRHs than those by the MLAD. The smaller deviations between the predicted and observed DRHs for the MRNG indicate that the MRNG is better than the MLAD in its capability 
to predict the DRHs for other storms. The computation time for the MRNG is somewhat longer than that for the MLAD. The UH from the MSAD model was found to perform better than the MRNG in predicting the DRHs but require much longer computation time. The results from the numerical investigation provide useful information to hydrologists in selecting an LP formulation.

Interestingly, the numerical results indicate that the MWSAD formulation with a negative $\alpha$ was the best in predicting the DRHs among all LP formulations. The statistical cross-validation method was incorporated into the framework of golden section technique to determine the optimal $\alpha$ in the weighing function for a selected error criterion. It was found that there exists a negative optimal $\alpha$ for each of the selected three error criteria. This observation implies that emphasizing fitting on peak discharge of DRH does not necessarily produce a UH equipped with a better predictive capability for future storm events. Application of the proposed MWSAD and MRNG formulations in this study show some promising features for the derived UHs. More extensive investigations using storms occurred in other watersheds is recommended for future research and application.

\section{Acknowledgement}

The authors are grateful to the anonymous reviewers for their useful comments.

\section{References}

Barnes, B.S., 1959, Consistency in unit hydrographs, Proc. ASCE 85(HY8), 39-63.

Bender, D. L. and Roberson, J. A., 1961, The use of a dimensionless unit hydrograph to derive unit hydrographs for some Pacific northwest basins, J. Geogr. Res. AGU 66, 521-527.

Björck, A., 1990, Least squares methods, in P. G. Ciarlet and J. L. Lions (eds), Handbook of Numerical Analysis Vol. 1, Elsevier, Amsterdam, pp. 467-652.

Bree, T., 1978, The stability of parameter estimation in the general linear model, J. Hydrology 37, 47-66.

Bruen, M. and Dooge, J. C. I., 1984, An efficient and robust method for estimating unit hydrograph ordinates, J. Hydrology 70, 1-24.

Chow, V. T., Maidment, D. R., and Mays, L. W., 1988, Applied Hydrology, McGraw-Hill, New York.

Collins, W. T., 1939, Runoff distribution graphs from precipitation occurring in more than one time unit, Civil Eng. ASCE 9(9), 559-561.

Deininger, R. A., 1969, Linear program for hydrologic analysis, Water Resour. Res. AGU 5(5), 1105-1109.

Dooge, J. C. I., 1965, Analysis of linear systems by means of Laguerre functions, J. SIAM Control Ser. A. 2(3), 396-408.

Dooge, J. C. I. and Garvey, B. J., 1978, The use of Meixner functions in the identification of heavily-damped systems, Proc. R. Irish Acad. A 78(18), 157-179.

Eagleson, P. S., Mejia-R, R., and March, F., 1966, Computation of optimum realizable unit hydrographs, Water Resour. Res. AGU 2(4), 755-764.

Grace, A., 1993, Optimization Toolbox, The Mathworks, Inc., South Natick, MA.

Kuchment, L. S., 1967, Solution of inverse flow problems for linear flow models, Soviet Hydrol. Sel. Pap. 2, 194-199.

Mays, L. W. and Coles, L., 1980, Optimization of unit hydrograph determination, J. Hydraul. Div. 106(HY1), 85-97.

Mays, L. W. and Tung, Y. K., 1992, Hydrosystems Engineering and Management, McGraw-Hill, New York. 
Morel Seytoux, H. J., 1982, Optimization methods in rainfall-runoff modeling, in V. P. Singh (ed.), Rainfall-Runoff Relationship, Water Resource Publications, Littleton, CO, pp. 487-506.

Newton, D. W. and Vinyard, J. W., 1967, Computer-determined unit hydrographs from floods, $J$. Hydraul. Div. ASCE 93(HY5), 219-234.

- O'Donnel, T. M., 1960, Unit hydrograph derivation by harmonic analysis, IAHS Publ. 51, 546-557.

Sherman, L. K., 1932, Stream flow from rainfall by unit-graph method, Engineering News Record 108, 501-505.

Singh, K. P., 1976, Unit hydrography - a comparative study, Water Resour. Bull. AWRA 12(2), 381-392.

Singh, V. P., 1988, Hydrologic Systems, Vol. I, Prentice-Hall, Englewood Cliff, NJ.

Snyder, W. M., 1955, Hydrograph analysis by the method of least squares, Proc. ASCE 81, 1-24.

The Mathworks, Inc., 1989, PC-MATLAB, User's Guide, South Natick, MA. 